\title{
Is a Virtual Reality-based Laboratory Experience a Viable Alternative to the Real Thing?
}

James R. McCusker Ph.D., Wentworth Institute of Technology

James R. McCusker is an Associate Professor at Wentworth Institute of Technology in the Department of Electrical Engineering. Since joining Wentworth in 2010, he has been heavily involved with an array of interdisciplinary design courses that range from introductory to capstone courses.

Mr. Mohammed A. Almaghrabi, Wentworth Institute of Technology

Mohammed A. Almaghrabi is a Trainee Engineer at ASM Process Automation, where he helps developing factory automation solutions for clients. He graduated from the University of the Wentworth Institute of Technology, with bechelors degree in Electromechanical Engineering in 2017.

Mr. Bryon Kucharski, Wentworth Institute of Technology

Student at Wentworth Institute of Technology 


\title{
Is a Virtual Reality-Based Laboratory Experience a Viable Alternative to the Real Thing?
}

\author{
James McCusker*, Mohammed A. Almaghrabi ${ }^{\dagger}$, Bryon Kucharski* \\ \{mccuskerj, almagharabim, kucharskib\}@ wit.edu \\ * Department of Electrical Engineering and Technology \\ ${ }^{\dagger}$ Department of Interdisciplinary Engineering \\ Wentworth Institute of Technology
}

\begin{abstract}
Laboratory practice is an important tool in the education of engineering students. The practical aspect of a laboratory-based education provides a tactile experience for students that allows students an opportunity to understand concepts and also prepare them for the engineering job market. One of the challenges faced by many institutions is that laboratory equipment and machines are costly and, as a result, a viable alternative option should be sought. This necessitates the adoption of virtual reality-based laboratory experiments that provide a laboratory-based instructional experience, similar to that of a real laboratory, yet utilizes a three-dimensional virtual simulation. This provides students with an opportunity to carry out experiments virtually and achieve instant results. In this study, a practical laboratory experience is provided through the use of a virtual reality simulation that is based on a real laboratory. Students are familiarized with the equipment through the provision of the real-world electronic bench equipment and simple electronic components resembling the real laboratory environment. The effectiveness of the virtual reality laboratory is studied using three sets of engineering students. The sets of students include those who have engaged in an actual laboratory experience, those who have experienced only the virtual reality-based laboratory environment, and a third group who has experienced both the real and the virtual reality laboratories. Using a common quiz, each set of students is assessed in their ability to identify and describe the uses of various laboratory equipment. Through the assessment, the viability of the virtual reality-based laboratory is studied regarding the effectiveness of it as an education tool. Recommendations are also made for institutions interested in designing similar experiences.
\end{abstract}

\section{Introduction}

Engineering education requires the transmission of information that provides students with an understanding of physical phenomena. Traditionally, engineering education utilizes laboratory 
experimentation to reinforce the learning experience ${ }^{1}$. As such, laboratories are an embedded part of scientific teaching as a whole ${ }^{2}$. Research indicates that involving students in hands-on activities can enhance STEM education and the overall quality of the learning experience ${ }^{3}$. STEM education exposes students to explore complex topics that can be reinforced through a laboratory experience. A positive hands-on laboratory experience can also have a significant impact on retention in engineering students. Knight et al. identified an over $15 \%$ increase in retention when first year engineering students are exposed to a hands-on project-based curriculum $^{4}$. Although the benefits of a hands-on laboratory experience are apparent, the significant cost of laboratory equipment can be prohibitive for some institutions ${ }^{2}$.

Computer simulation has been shown to be an effective tool that can be a viable, albeit lower cost, alternative to the traditional hands-on laboratory experience. Simulations have been shown to be an effective tool in enhancing traditional instruction ${ }^{5}$ and they also provide a degree of flexibility in instructing material ${ }^{6}$. They also allow for a structured method to improve understanding while working in a safe environment wherein students can explore without the possibility of damaging equipment or risking potential injury ${ }^{7}$. However, the inability to physically work with the real equipment has shown to be a limiting factor for introductory-level students and it is more beneficial for students that have prior experience with the laboratory hardware ${ }^{8}$. The benefit of simulation-based laboratory instruction is more pronounced when it is used in conjunction with the traditional hands-on approach ${ }^{5}$. It has been show that computer simulation can be effectively utilized to improve understanding in preparation for a traditional laboratory experience ${ }^{1,5}$. This combined approach can be an effective method to both improve safety and develop a degree of familiarity with the laboratory equipment prior to entering the actual laboratory.

While simulation can be an effective tool as a supplement to the traditional laboratory experience, it has been shown that games provide a positive impact on the effectiveness of education over that of simulation ${ }^{9}$. Through the use of intelligently designed games, students are more apt to engage the material and improve their understanding. However, the effectiveness of games as a learning tool is heavily influenced by the pedogeological context ${ }^{10}$ and can be enhanced by exploring additional mediums for providing the content. Over the last two decades, the virtual reality community has been moving from interactive 3-D simulations to implementing games to improve the delivery of the content ${ }^{11}$. Virtual Reality presents a compelling medium that provides a user with a feeling of being present in another environment ${ }^{12}$. As an educational tool, virtual reality has been show to be an effective tool in both K-12 and higher education ${ }^{9}$. In addition, it has been shown that games based in virtual reality can improve the learning experience in users ${ }^{13}$.

This study explores the viability of a virtual reality-based laboratory environment as an alternative to the traditional hands-on lab experience. For this, the Virtual Electronics Laboratory is developed and deployed to first-year students at the College of Engineering and Technology at Wentworth Institute of Technology in Boston, Massachusetts. The effectiveness of this delivery, as compared to the traditional approach, is evaluated with a standardized quiz that was deployed to all students that participated in the study. 


\section{Project Description}

The Virtual Electronics Laboratory was modeled to emulate the actual laboratory environment that students utilize in the electronics Laboratories at Wentworth Institute of Technology. To provide a realistic laboratory experience, the HTC Vive Virtual Reality System was utilized for the delivery of the content. This system allows for separate projections to each of the user's eyes which provides the perception of a 3-dimensional environment. The system also allows for a room-scale virtual reality experience. This feature allows for a user to walk freely around the Virtual Electronics Laboratory that is designed to provide an accurate representation of the true electronics laboratory.

The Virtual Electronics Laboratory environment was constructed using the Unity Game Engine ${ }^{14}$ which provides an ideal platform for developing virtual reality applications. The various models that populate the electronics lab were generated using an array of tools. Many of the objects were developed using Blender ${ }^{15}$ in conjunction with Solidworks. First, the 3D models were developed using Solidworks or imported from existing models available from the online model depository GrabCAD ${ }^{16}$. The models were then imported to Blender to provide textures and make minor modifications to provide a realistic representation of the true instruments.

For complex geometries, other methodologies could be employed that utilize various imaging and scanning hardware. For modeling 3-dimensional objects, the Microsoft Kinect may be used as a 3D scanner that is capable of mapping the object acquired from the IR camera and then provide photo-realistic textures that are acquired by the RGB camera. This is a low-cost solution that provided an adequate platform for developing these models with a simple-to-use SDK. For three dimensional environments, the ZED Stereo vision camera provides an effective tool wherein a detailed environment can be constructed.

The bench-top equipment that students utilize in the electronics laboratories in the Electrical and Computer Engineering Department are as follows:

- Hewlett Packard E3621A triple output DC power supply

- Agilent 33521A Function/waveform Generator

- Agilent 34461A Digital Multimeter

- Keysight 4 channel Oscilloscope

All of this equipment, as well as other related components, such as an Arduino microcontroller and a breadboard, were constructed using the previously described methods and imported into the Unity Engine. Once there, rules were applied that allow a user to interact with the Virtual Electronics Laboratory. These rules allow a user to pick up various objects, access information menus, and walk around the laboratory. Students access information regarding the laboratory equipment with the HTC Vive controllers by colliding the laser pointer with 3D objects. Once a collision occurs, the user is prompted with a window with a description of the 3D object. The window appears at eye-level with the user, but at a distance far enough away to read the text. The window provides the user the option to read the text description or have the text read back through the computer speakers. A general framework was created via a script to make it easy to modify 
the details of the 3D object such as the name and description. Once these rules were applied, the Lab was deployed as an application that can be accessed through the HTC Vive virtual reality system.

\section{Effectiveness Study}

This study involves first year students enrolled in the College of Engineering at Wentworth Institute of Technology. Students involved in this study are drawn from the Biomedical Engineering, Biological Engineering, Computer Science, Computer Engineering, Civil Engineering, Electrical Engineering, Electromechanical Engineering, and Mechanical Engineering majors within the University. The focus of the lab experience is on developing a fundamental understanding of the identification and use of basic laboratory equipment that are utilized in the Electrical and Computer Engineering Department.

\subsection{Study Participants}

There were 45 participants that completed all required aspects of the study. Of these participants, 18 utilized the Virtual Electronics Laboratory and 27 engaged in only the Traditional Laboratory Experience. The evaluation involves students that are introduced to the laboratory equipment using the traditional hands on approach, those that are using a purely virtual reality-based approach, and those that are presented with both the traditional approach in conjunction with the virtual reality-based approach. Each of these groups is described as the following:

- Traditional Laboratory Experience ( $N=27)$ : The students that comprise the Traditional Laboratory Experience group engage in 4 2-hour lab sessions utilizing the the equipment shown in Figure 1. Through these 4 laboratory sessions, students work in groups of 2 or 3 to build basic circuits on a breadboard, program Arduino microcontrollers, and perform testing using a bench-top DC power supply, function generator, digital multimeter, and oscilloscope. After completing the laboratory experiments, students complete a group lab report.

- Virtual Reality Laboratory Experience $(N=9)$ : The students that comprise the Virtual Reality Laboratory Experience group engage in one 15-30 minute session in the Virtual Electronics Laboratory (Figure 2). The participants who tested the virtual reality lab were provided a tutorial before exploring the virtual lab. The tutorial informed the students in the safety considerations for the use of a virtual reality system and in the methods of utilizing the controllers to navigate and interact their environment.

Once the tutorial was completed, the students were allowed to engage in the Virtual Electronics Laboratory. In the Virtual Electronics Laboratory trial, students could explore and manipulate the various equipment. Students were allowed to pick up any of the various components, activate informational displays and voice overs, and gain an overall familiarity with the electrical lab. Once students felt they had fully explored the lab, they concluded their session.

- Traditional and Virtual Reality Experience (Combined) (N=9): The students that comprised the Combined experience group engage first in the Traditional Laboratory Experience 


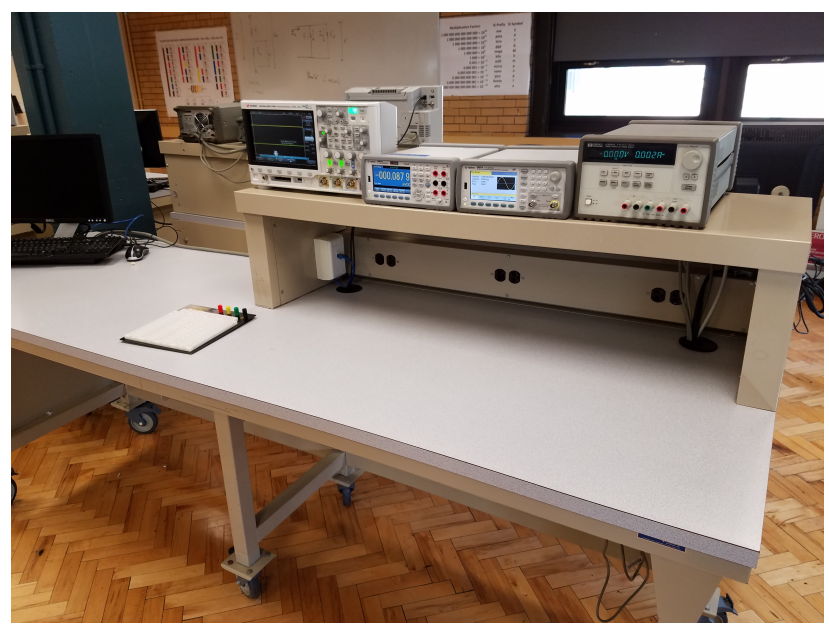

Figure 1: The Actual Laboratory Environment

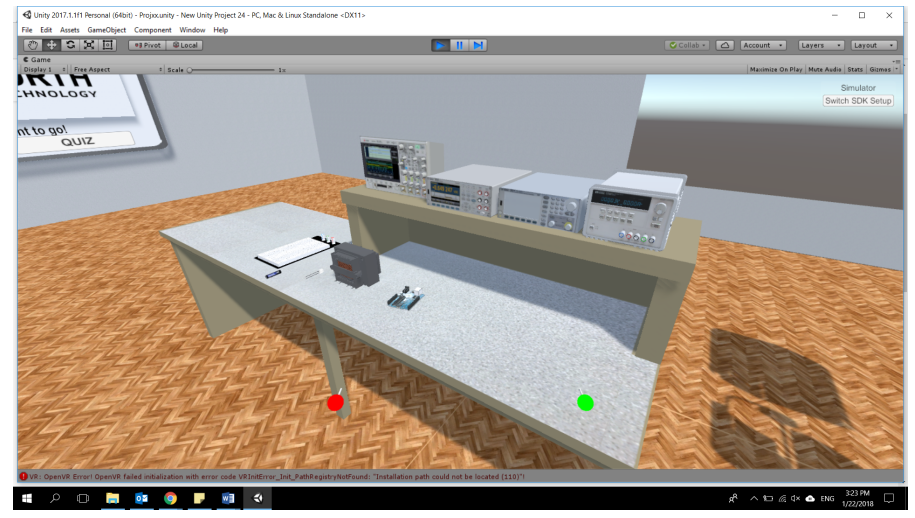

Figure 2: The Virtual Reality Laboratory Environment

described above. Following that, they supplement their understanding by engaging in the Virtual Electronics Laboratory.

\subsection{Student Evaluation Procedure}

All students were evaluated in their familiarity and understanding of the use of the laboratory equipment through an online quiz that was delivered through the learning management system Blackboard. After completion of the assigned lab experience(s), students were instructed to complete the quiz component prior to the conclusion of the study. The date set for the conclusion of the study was the same for all participants, regardless of when they completed their assigned experience. This quiz was comprised of ten questions that could be broken into two categories; four identification questions and six use questions. The identification questions provided an image of each of the pieces of bench-top equipment and required that the student identifies the appropriate title from a list of options (Figure 3). The use questions required that students associate each of the pieces of equipment with with the appropriate use, function, or purpose from a list of options (Figure 4). 


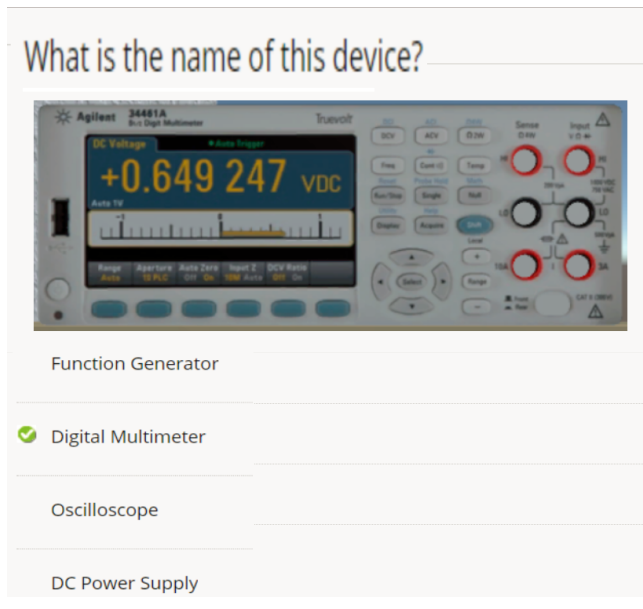

Figure 3: Sample Identification Question

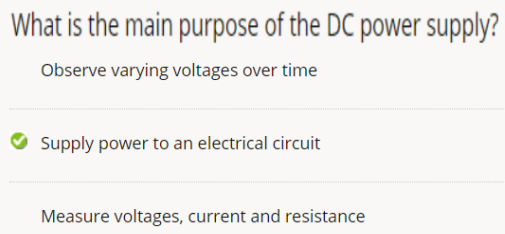

Figure 4: Sample Use Question

Evaluation also solicited feedback from each of the Virtual Reality Laboratory Experience participants immediately following their session. The feedback focused on usability and recommendations for future improvements.

\section{Student Evaluation Results}

The average score for all students in each of the student participation groups identified in Section 3.1 is shown in Figure 5.

Here, we can observe that the traditional laboratory experience provides a more effective understanding of the equipment over that of a Virtual Electronics Laboratory. The average overall score for students that experience the virtual lab as the only means of delivery of the content averaged a score of $69.8 \%$. In comparison, the students that experienced only the traditionally delivered electronics lab achieved an average score of $82.4 \%$. However, in a two-tailed t-test, the difference could not be shown to be statistically significant to a $95 \%$ confidence factor $(\mathrm{p}=$ 0.0952). When comparing the combined delivery to that of the virtual reality alone, we identify a marked improvement with an average score of $91.8 \%$ that is statistically significant with a p-value of 0.0105 .

The results from the separated questions sets allow for additional insight into the potential 


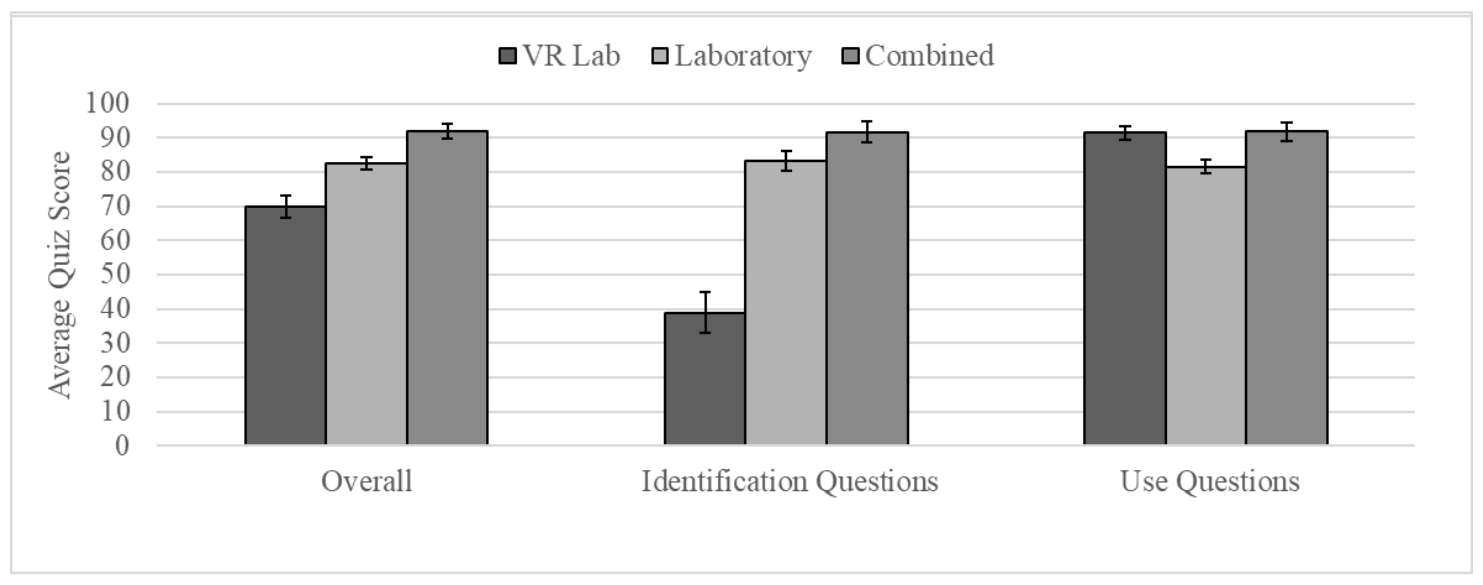

Figure 5: Assessment Quiz Results (error bars +/- 1 standard error)

effectiveness of a virtual delivery of laboratory content. For the equipment identification question set, the students that were delivered the content virtually performed significantly worse than those that were delivered the content using the traditional or combined approach. In this, only one of nine students successfully identifying all four pieces of bench-top equipment and three were unable to successfully identify any of the equipment. In comparison, seven of the nine students that experience the combined delivery successfully identified all four pieces of equipment.

For the use questions, the virtual electronics lab students performed significantly better than they did on the identification questions with an average score of $91.4 \%$ which almost matched that of the combined delivery students which averaged $91.8 \%$. In comparison, the students that experienced the traditionally delivered lab content averaged an $81.49 \%$. Although not statistically significant $(\mathrm{p}=0.1795)$, this does indicate the potential for content delivered through virtual reality to have benefits over that of traditionally delivered content.

Regardless of the question set (identification, use, and overall), it can be seen that the combined delivery produced better results than all other delivery methods. This indicates that combining a virtual delivery of content with that of the traditional approach can enhance the effectiveness of the overall understanding of the laboratory environment and the overall learning experience. This observation is echoed in the literature pertaining to simulation-based laboratory experiences. Olin et.al were able to identify that simulations in conjunction with the traditional delivery produced a higher level of understanding than that of the Traditional approach alone ${ }^{6}$. However, this particular study did not consider the use of virtual reality as the means of delivery. Since the results of our study could not illustrate a statistically significant improvement of a combined lab over that of traditionally delivered content, further exploration is necessary. 


\section{Discussion}

The results of this study illustrate the promise of a virtual realty-based delivery method. This work illustrates the first phase of the development of the Virtual Electronics Laboratory and there are some considerations to be taken into account for this study. First of all, there are a few areas of improvement for the software to enhance the effectiveness of it as a learning tool.

- General software improvements: Feedback from users indicated there were some limitations in the delivery of text-based content. Some users indicated a sensitivity to the text size and color of the pop-up windows that provided the information regarding the equipment. Additionally, the content of the pop-up windows was lengthy and often difficult to absorb. Further experimentation with text size and color as well as content must be done to improve the overall experience.

- Expanded capabilities: Currently the Virtual Electronics Lab is almost entirely an environment for users to explore. However, to improve the capability of the software, electronics circuit simulations are being developed to allow students to build and test circuits using the virtual bench-top equipment. Although similar software has been developed as a standalone java-based simulation ${ }^{7}$, allowing this capability in virtual reality could greatly enhance the capabilities of the Virtual Electronics Laboratory.

- Expand to other laboratory environments: Although the Virtual Electronics Laboratory is modeled after the actual electronics laboratory at the Evaluated University, the tools used to construct it in Unity can be applied to other laboratory environments. The use of the Zed stereo vision camera and Microsoft Kinect allow for quick integration into the virtual realm and other laboratories. Currently the development of a Virtual Strengths of Materials Laboratory and Motors Laboratory is being investigated for future development.

Beyond the improvements that can be made to the Virtual Electronics Laboratory, there are other things to consider in the development of similar virtual laboratories.

- Effectiveness of Virtual Reality-Based Laboratories: Although Virtual Reality as a medium presents exciting opportunities, it has yet to illustrate that it has a marked improvement over that of the traditional approach. Integrating games and realistic simulations into the instruction can be a significant undertaking, however this may allow for a drastic improvement in the effectiveness of this as a learning tool.

- Over-saturation: It has been shown that instruction through simulation and other electronic mediums can lead to over-saturation that limits the effectiveness ${ }^{9}$. With this in consideration, it is noted that an overreliance on these mediums can be detrimental to the educational process. Therefore, the designers of these virtual environments should take into consideration the length and repetitiveness of a virtual lab session.

- Safety: In the virtual realm, students can explore and make mistakes without damaging equipment or risking personal injury. Although respect for equipment is necessary for professional practice, students can be effectively introduced to the proper use of equipment prior to stepping foot in an actual lab. Although virtual reality-based laboratory environments can be used to improve safety when students move to a physical laboratory, it 
must also be noted that prolonged exposure to virtual reality using currently available systems can have health effects to a user. Therefore this must be taken into consideration when designing virtual lab environments and activities.

- Opportunities present in Virtual Reality: Virtual Reality-based instruction can present opportunities that are not present in a traditional laboratory environment. For example, this medium allows for the potential development of software to illustrate current flowing though a circuit that a student constructs on a breadboard to enhance understanding. Similarly, in the event that a student makes an improper measurement or places themselves at potential risk, warnings could be provided to improve understanding and also prevent potentially unsafe laboratory practice.

\section{Conclusions}

The Virtual Electronics Laboratory presents the first phase of an initiative to develop realistic laboratory experiences that could both supplement the traditional laboratory experience and potentially serve as a viable cost effective alternative. Assessment indicates that, although it is not currently as effective as the traditional hands-on approach overall, there are areas that a virtual laboratory environment may be able to exceed the traditional approach in educational effectiveness. Although limitations exist with this medium, future improvements in virtual reality hardware and software present exciting opportunities for future development.

\section{Acknowledgements}

The authors would like to acknowledge the H.C. Lord Professorship Fund for funding this research. We would also like to thank Dr. Aaron Carpenter, Tory Lam, and the Accelerate program at Wentworth Institute of Technology for their support throughout the development of this work. 


\section{References}

[1] L.D. Feisel and A.J. Rosa. The role of the laboratory in undergraduate engineering education. ASEE Journal of Engineering Education, 94(1):121-130, 2005.

[2] R.T. White. The link between the laboratory and learning. International Journal of Science Education, 18(7): 761-774, 1996.

[3] C. Xianglei. Stem attrition: College students' paths into and out of stem fields. statistical analysis report. nces 2014-001. National Center for Education Statistics, 2013.

[4] D.W Knight, L.E. Carlson, and J.F Sullivan. Staying in engineering: Impact of a hands-on, team-based, first-year projects course on student retention. In 2003 ASEE Annual Conference \& Exposition, June 2003.

[5] N. Rutten, W.R van Joolingen, and J.T. van der Veen. The learning effects of computer simulations in science education. Computers and Education, 58(1):136-153, 2012.

[6] J. Olin Campbell, J.R. Bourne, P.T. Mosterman, and A.J Brodersen. The effectiveness of learning simulations for electronic laboratories. ASEE Journal of Engineering Education, 91(1):81-87, 2002.

[7] H. Hodge, S.H. Hinton, and M. Lightner. Virtual circuit laboratory. ASEE Journal of Engineering Education, 90(1):507-511, 2001.

[8] C. Lemckert and J. Florance. Real-time internet mediated laboratory experiments for distance education students. British Journal of Educational Technology, 33(1):99-103, 2002.

[9] Z. Merchanta, E.T. Goetz, L. Cifuentes, W. Keeney-Kennicutt, and T.J.Davisa. Effectiveness of virtual reality-based instruction on students' learning outcomes in k-12 and higher education: A meta-analysis. Computers and Education, 70:29-40, 2014.

[10] S. Chatterjee, A. Mohanty, and B. Bhattcharya. On the exploration of game-based learning in stem education with the development and application of virtual reality course modules. In 2011 IEEE International Conference on Technology for Education, July 2011.

[11] M. Zyda. From visual simulation to virtual reality to games. Computer, 38(9):25-32, 2005.

[12] J.M Loomis. Presence in virtual reality and everyday life: Immersion within a world of representation. Presence: Teleoperations and Virtual Environments, 25(2):169-174, 2016.

[13] L. Zhang, B.E. Whiteley, D.K. Sharma, I.K. Dabipi, W.J. Brown, W. Zhu, and X. Tan. On the exploration of game-based learning in stem education with the development and application of virtual reality course modules. In 2017 ASEE Annual Conference \& Exposition, June 2017.

[14] Unity game engine. https://unity3d.com/.

[15] Blender. https://www.blender.org/.

[16] Grabcad. https://grabcad.com/. 